\title{
Post-Harvest Alteration of the Main Chemical Ingredients in Ligusticum chuanxiong HoRT. (Rhizoma Chuanxiong)
}

\author{
Song-Lin LI, ${ }^{a}$ Ru YAN, ${ }^{a}$ Yun-Kau TAM, ${ }^{b}$ and Ge LIN ${ }^{*}, a$ \\ ${ }^{a}$ Department of Pharmacology, Faculty of Medicine, The Chinese University of Hong Kong; Hong Kong, SAR: and \\ ${ }^{b}$ SinoVeda Ltd.; Hong Kong, SAR. Received August 29, 2006; accepted October 17, 2006
}

\begin{abstract}
Rhizoma Chuanxiong (Ligusticum chuanxiong HoRT.) is a commonly used traditional Chinese medicinal herb for the treatment of cardiovascular disorders. Significant variations of the main components in this herb were observed in commercial samples. The present study investigated effects of post-harvest drying and processing methods on nine main components in the herb. Results showed that drying at $60^{\circ} \mathrm{C}$ or under the sun the contents of three major constituents, namely senkyunolide A (4), coniferylferulate (5) and $Z$-ligustilide (6), decreased significantly, while the contents of ferulic acid (1), riligustilide (8) and levistolide $A$ (9) increased significantly. Senkyunolide I (2) and senkyunolide $H(3)$, which were not detected in fresh herbs, appeared in dried samples. Similar chemical alterations, such as decrease in the contents of three major ingredients and increase in the contents of compounds $1,2,3,8$ and 9 , were also observed in differently processed herbal samples. The possible converting mechanisms of these components were clarified by employing pure major components treated under the same conditions.
\end{abstract}

Key words Ligusticum chuanxiong; phthalides; post-harvest chemical alteration; processing

Rhizoma Chuanxiong (Chinese name Chuanxiong), the dried rhizome of Ligusticum chuanxiong HoRT. (Umbelliferae), is one of the most commonly prescribed traditional Chinese medicinal (TCM) herbs for the treatment of cerebroand cardio-vascular diseases. Various chemical constituents have been identified in this herb, ${ }^{1-3)}$ and several components, including ferulic acid (1), senkyunolide A (4), Z-ligustilide (6), 3-butylidenephthalide (7), riligustilide (8) and levistolide A (9) (Fig. 1), have been reported to be the active components contributing to the therapeutic effects of Chuanxiong. ${ }^{4-9)}$

As a series of systematic investigation and development of quality control of Chuanxiong herb, our research team had determined main ingredients in dried herbs purchased from different herbal stores in Hong Kong and Mainland China as well as in fresh rhizomes grown in Sichuan Province, China. In all samples tested, senkyunolide A (4), coniferylferulate (5) and $Z$-ligustilide (6) were determined as the three major components, whereas ferulic acid (1) and 3-butylidenephthalide (7) were found with relatively lower contents. Interestingly, senkyunolide I (2) and senkyunolide H (3) were only detected in the dried samples. Furthermore, riligustilide (8) and levistolide A (9) were found in all dried samples but only some of the fresh rhizomes with significantly lower quantities. The results demonstrating significant differences in chemical profiles between fresh and dried Chuanxiong herbs have been previously published. $1,3,10,11)$

In TCM practice, harvested plants usually have to be dried and/or processed prior to prescription. Drying and processing methods vary in different places of China and are often based on traditional knowledge. However, they lack well-controlled and standardized procedures. Specifically described processing procedures are documented for only about $4 \%$ of all processed TCM herbs according to China Pharmacopoeia. ${ }^{12)}$ The most common procedures for processing Chuanxiong include stir-frying alone and frying with different solid or liquid adjuvants. The most commonly used adjuvants are bran and wine. ${ }^{13)}$ On the other hand, sun-drying or heating in oven are the most common and traditional ways for drying Chuanxiong. It has been reported that some of the constituents in Chuanxiong, e.g., 4, 5 and $\mathbf{6}$ are photo- and/or

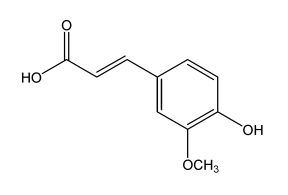

1

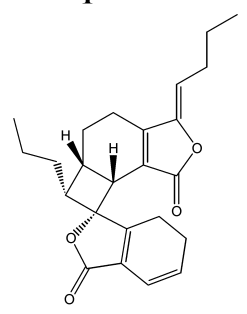

8

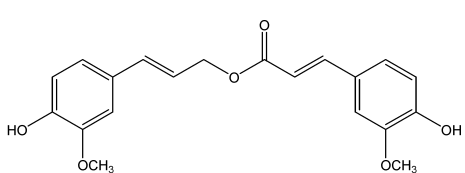

5

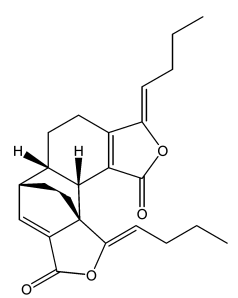

9

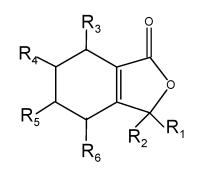

\begin{tabular}{|c|c|c|c|c|c|c|}
\hline & $R_{1}$ & $\mathrm{R}_{2}$ & $\mathrm{R}_{3}$ & $\mathrm{R}_{4}$ & $\mathrm{R}_{5}$ & $R_{6}$ \\
\hline 2 & & & $\beta-\mathrm{OH}$ & $\alpha-\mathrm{OH}$ & $\mathrm{H}$ & $\mathrm{H}$ \\
\hline 3 & & & $\beta-\mathrm{OH}$ & $\beta-\mathrm{OH}$ & $\mathrm{H}$ & $\mathrm{H}$ \\
\hline 4 & & $\mathrm{H}$ & & & & \\
\hline 6 & & & & & $\mathrm{H}$ & $\mathrm{H}$ \\
\hline 7 & & & & & & \\
\hline
\end{tabular}

Fig. 1. Chemical Structures of Nine Main Components of Rhizoma Chuanxiong

1: ferulic acid, 2: senkyunolide I, 3: senkyunolide $H, 4$ : senkyunolide A, 5: coniferylferulate, 6: $Z$-ligustilide, 7: 3-butylidenephthalide, 8: riligustilide, 9: levistolide A. 


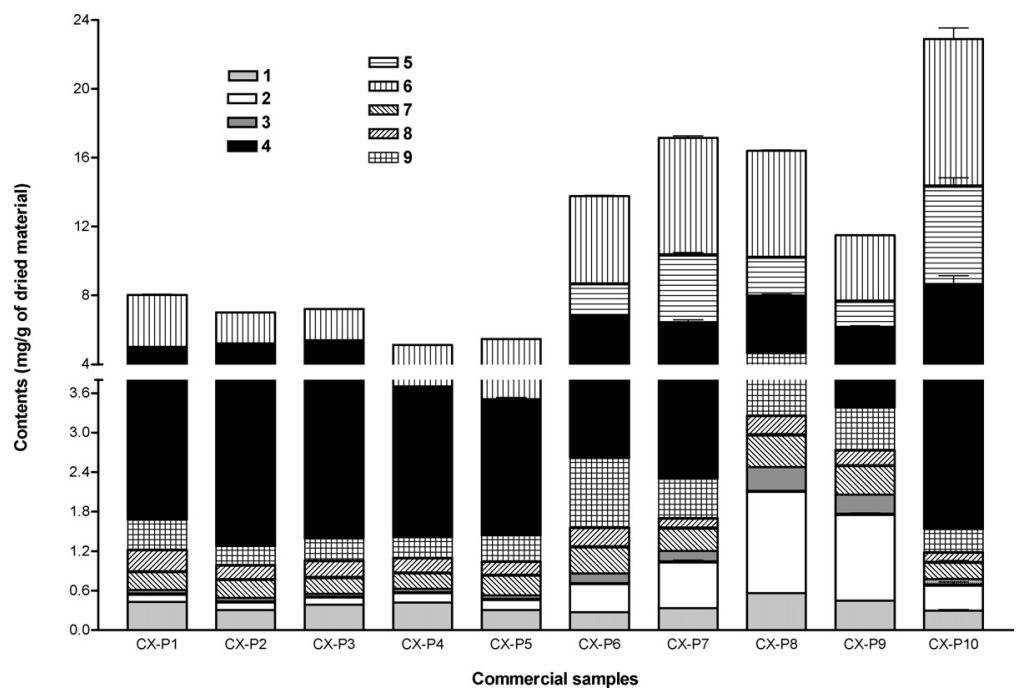

Fig. 2. Contents of Main Components in Ten Commercially Available Rhizoma Chuanxiong Herbs Processed Using Different Methods

thermo-labile, ${ }^{14-18)}$ although data about the influence of post-harvest drying and processing on chemical profiles of Chuanxiong are unavailable. With unavailability of detailed processing information on commercial samples, our preliminary evaluation of ten purchased Chuanxiong herbs, which were processed differently, demonstrated significant variations in chemical profiles (Fig. 2). Therefore, the present study investigates the chemical alterations of the main ingredients in Chuanxiong herbs caused by different drying and processing methods.

\section{Experimental}

Chemicals and Materials Compounds 1 (Acros Organics, Geel, Belgium), 6 (ChromaDex, Santa Ana, CA, U.S.A.) and 7 (Aldrich Chemical Company, St. Louis, MO, U.S.A.) were purchased. Compounds 2, 3, 4, 5, 8 and 9 were isolated in our laboratories. Confirmation of identities and purities of the isolated compounds has been reported previously. ${ }^{1,3,10,11)}$ Purities of all commercial and isolated compounds were higher than $95 \%$.

Fresh rhizomes of Ligusticum chuanxiong HoRT. (CX-F) were collected from a Chuanxiong cultivating base of Tianfu Chuanxiong Pharmaceutical Development Company Ltd. in Dujiangyan County, Sichuan Province, China on 20 May 2003 (a traditional harvesting time for Chuanxiong). ${ }^{11}$ The fresh samples were stored in a dark place at $-20^{\circ} \mathrm{C}$. Processed Chuanxiong herbs were purchased from local herbal stores in Hong Kong (CX-P1: form Hop Tak Luing, CX-P2 and CX-P3: Hip Wo, CX-P4: Kwong Yuen Tai, CX-P5: Yung Tung, CX-P6: Eu Yan Sang) and Mainland China (CX-P7: Dujiangyan, Sichuan, CX-P8: Pengzhou, Sichuan: CX-P9: Chengdu, Sichuan, CX-P10: Nanjing, Jiangsu). However, as a normal practice, detailed information on processing methods of these commercial samples was unavailable. The voucher specimens of all Chuanxiong samples were deposited at the Department of Pharmacology, the Chinese University of Hong Kong, and their identities were authenticated according to China Pharmacopoeia. ${ }^{12)}$

Treatment of Pure Compounds In order to evaluate stability of three major components $4-\mathbf{6}$ and $\mathbf{7}$, their isolated pure forms were treated under two different conditions. Condition B: the individual compound was kept in a capped amber glass vial and heated at $60^{\circ} \mathrm{C}$ for $12 \mathrm{~h}$ in an oven. Condition $\mathrm{C}$ : each compound was kept in a capped transparent glass vial and exposed to sunlight for $8 \mathrm{~h}$. Each compound under each condition was conducted in triplicate. The treated samples were analyzed by HPLC-DAD and the results were compared with the untreated pure sample (condition A) for the calculation of percentage degradation.

Drying and Processing Methods Fresh rhizomes (CX-F) were dried either directly or after cutting into slices via heating in an oven at $60^{\circ} \mathrm{C}$ for $10-24 \mathrm{~h}$ or exposed to sunlight for $2-8 \mathrm{~d}$ (Table 1). The oven dried rhizome slices (CX-D2) underwent further processing by three different methods: 1) Stir-frying alone Chuanxiong (CX-D2-P1): the dried slices were stir-
Table 1. Drying and Processing Conditions for Different Chuanxiong Samples

\begin{tabular}{|c|c|c|}
\hline Sample code & Sample prior to treatment & Drying/processing method \\
\hline $\begin{array}{l}\text { CX-D1 } \\
\text { CX-D2 } \\
\text { CX-D3 }\end{array}$ & $\begin{array}{l}\text { Slice of fresh sample (CX-F) } \\
(2 \mathrm{~mm} \text { in thickness })\end{array}$ & $\begin{array}{l}\text { Lyophilized in the dark } \\
\text { Dried at } 60^{\circ} \mathrm{C} \text { for } 10 \mathrm{~h} \text { in oven } \\
\text { Sun-dried for } 2 \mathrm{~d}\end{array}$ \\
\hline $\begin{array}{l}\text { CX-D4 } \\
\text { CX-D5 }\end{array}$ & $\begin{array}{l}\text { Rhizome of fresh sample } \\
\quad(\mathrm{CX}-\mathrm{F})\end{array}$ & $\begin{array}{l}\text { Dried at } 60^{\circ} \mathrm{C} \text { for } 24 \mathrm{~h} \text { in oven } \\
\text { Sun-dried for } 8 \mathrm{~d}\end{array}$ \\
\hline $\begin{array}{l}\text { CX-D2-P1 } \\
\text { CX-D2-P2 } \\
\text { CX-D2-P3 }\end{array}$ & $\begin{array}{l}\text { CX-D2 } \\
\text { CX-D2 } \\
\text { CX-D2 }\end{array}$ & $\begin{array}{l}\text { Stir-fried alone } \\
\text { Bran-fried } \\
\text { Wine-pretreated then stir-fried }\end{array}$ \\
\hline
\end{tabular}

fried alone in a pan until appearance of a yellowish colour; 2) Bran-frying Chuanxiong (CX-D2-P2): bran (18g) was added in a pre-heated pan and sautéed until the bran began to smoke, the dried slices $(100 \mathrm{~g})$ were then added and fried continuously until appearance of the yellowish colour. The resultant samples were cooled down to room temperature and the bran was filtered; and 3) Wine-pretreated stir-frying Chuanxiong (CX-D2-P3): the dried slices $(100 \mathrm{~g})$ were soaked with $10 \mathrm{ml}$ of rice wine $(17 \%$ alcohol). After wine was absorbed completely, slices were fried until appearance of a yellowish colour. The detailed sample codes, drying and processing conditions are given in Table 1.

Qualitative and Quantitative Determination The fresh Chuanxiong sample (CX-F) was cut into small pieces and lyophilized (CX-D1). The lyophilized sample, dried samples (CX-D2 to CX-D5), and processed samples (CX-D2-P1 to CX-D2-P3) were pulverized, extracted, and analyzed according to the procedures reported previously. ${ }^{3)}$ For pure compounds 4-7, each of the treated and non-treated samples was dissolved in methanol, filtered through a $0.45 \mu \mathrm{m}$ PTFE syringe filter, and then subjected to HPLC analysis. Each sample was analyzed in triplicate. Qualitative and quantitative determinations of compounds $\mathbf{1}-\mathbf{9}$ in various samples were performed by our previously developed HPLC-MS ${ }^{1)}$ and HPLC-DAD ${ }^{3)}$ methods, respectively.

Statistic Analysis Results were expressed as mean \pm standard deviation (S.D.). Values to be compared between fresh and dried herbs and between non-processed and processed herbs were evaluated using the Mann-Whitney test. Results were considered significant when the $p$ value was less than 0.05 .

\section{Results and Discussion}

Chemical Alteration of Compounds 4-7 under Various Conditions As illustrated in Fig. 3, percentages of the unchanged compounds and their corresponding degradation products were calculated based on $1: 1$ stoichiometric con- 


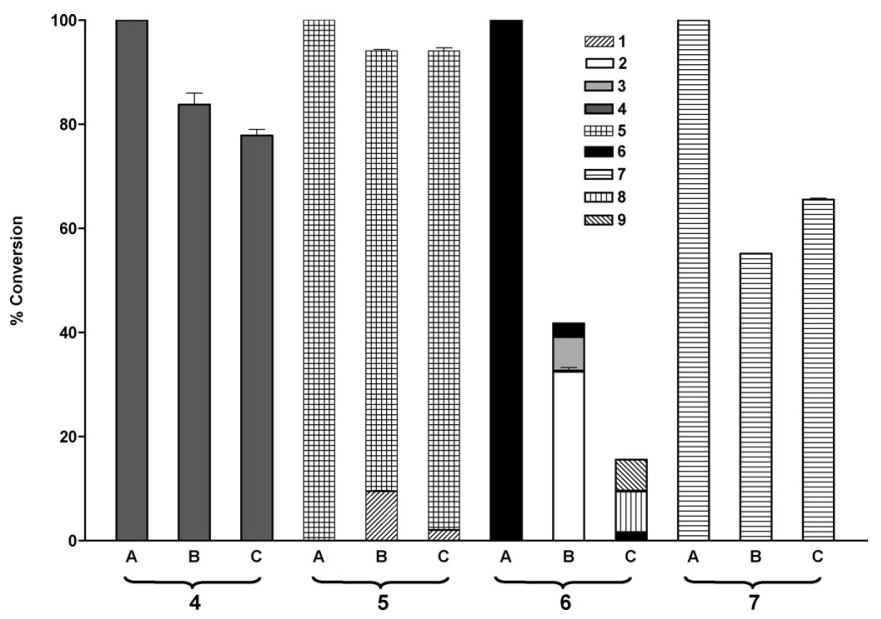

Fig. 3. Percentage of Degradation and Chemical Conversion of Compounds $4-7$

A: non-treated, B: $60^{\circ} \mathrm{C}$ for $12 \mathrm{~h}$ in oven, C: exposed to sunlight for $8 \mathrm{~h}$.

version. Under both conditions compound 5 decreased significantly to form $\mathbf{1}$ as its predominant degraded product. The results were in good agreement with previous reports in that $\mathbf{5}$ could be hydrolyzed to $\mathbf{1}$ under various conditions including high temperature. ${ }^{15-17)}$ In the case of compound $\mathbf{6}$, significant degradation occurred under both conditions although very low recovery was obtained. Different degradation products were identified under different conditions. At an elevated temperature in the dark, the main degradation products were hydroxylated compounds $2(31 \%)$ and $\mathbf{3}(6 \%)$. While under sunlight exposure, two dimerized compounds 8 $(8 \%)$ and $9(6 \%)$ were detected (Fig. 3). Similar observations were reported previously in that chemical conversions of $\mathbf{6}$ occurred when it was exposed to sunlight or kept at room temperature without any direct contact to sunlight, generating $\mathbf{8}$ and $\mathbf{9}$ or $\mathbf{2}$ and $\mathbf{3}$, respectively. However, the degradation products were not quantified in the study. ${ }^{14)}$ When $\mathbf{4}$ was heated at $60{ }^{\circ} \mathrm{C}$ or exposed to sunlight, about $15 \%$ or $22 \%$ degradation occurred, however the degradation products could not be identified. Furthermore, the stability of compound 7 was also evaluated because it is naturally present but not a degradation product of any of the major components in the herb. Treated at an elevated temperature or exposed to sunlight, 7 decomposed to $55 \%$ and $65 \%$, respectively. However, its degradation products could not be identified.

The results demonstrated that $\mathbf{4 - 7}$ were all thermo- and photo-unstable. At high temperature and/or under sunlight exposure, 6 and 7 significantly decomposed, while $\mathbf{4}$ and $\mathbf{5}$ degraded at a relatively slower rate. Furthermore, $\mathbf{5}$ was hydrolyzed to form $\mathbf{1}$ under both conditions, whereas $\mathbf{6}$ was thermolabile and photo-unstable, forming hydroxylated compounds $\mathbf{2}$ and $\mathbf{3}$ and dimerized compounds $\mathbf{8}$ and $\mathbf{9}$, respectively. The proposed chemical conversions of $\mathbf{5}$ and $\mathbf{6}$ are shown in Fig. 4.

Influences of Drying Methods on the Main Components in Chuanxiong The lyophilized sample (CX-D1) was used as the fresh control, because the main constituents in the fresh sample do not changed significantly when sample was dried at $0^{\circ} \mathrm{C}$. Chemical profiles of all dried herbs were markedly different from the fresh control, and several compounds, namely $2,3,8$ and 9 , which were not detected (or

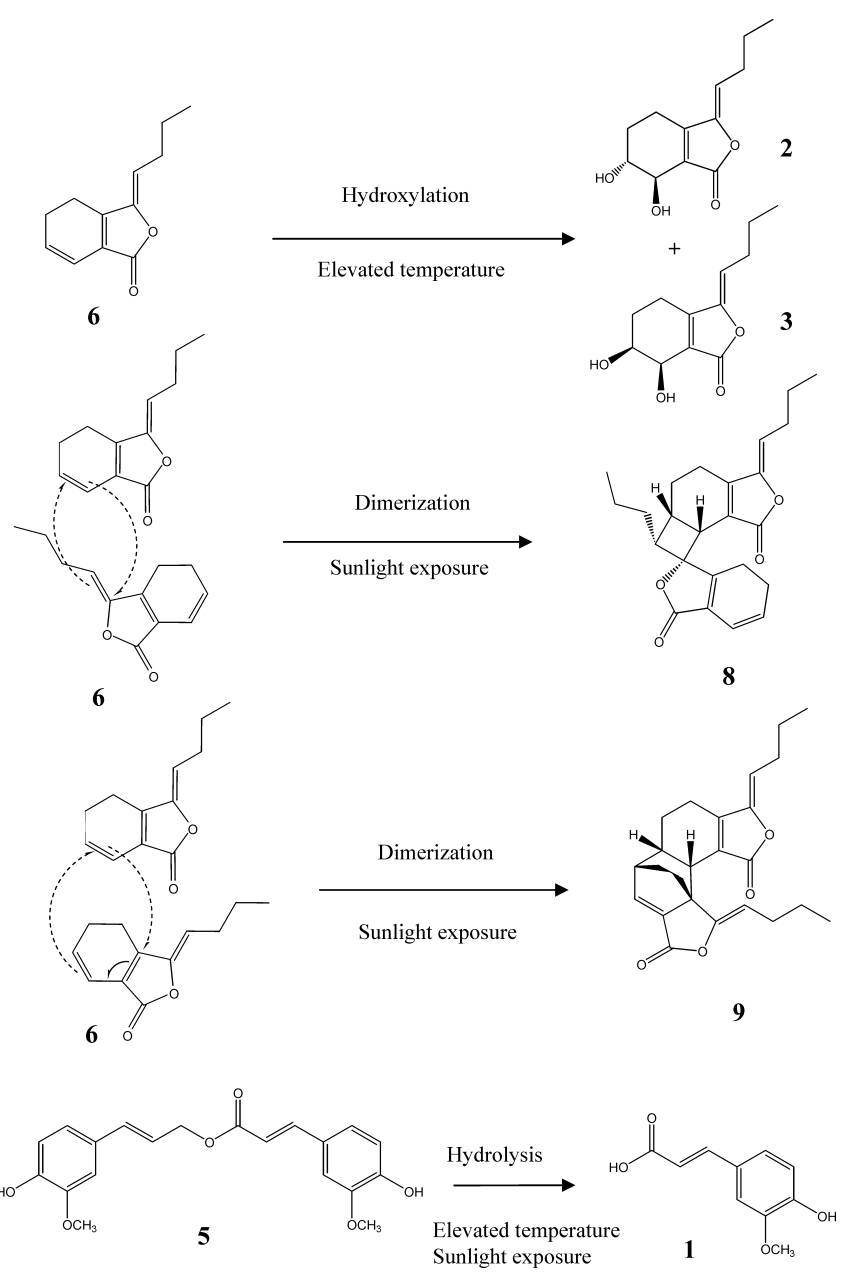

Fig. 4. Proposed Chemical Conversions of Compounds $\mathbf{5}$ and $\mathbf{6}$

their contents were bellow the detection limits) in fresh rhizome, appeared in dried samples (Fig. 5). Compared with the fresh control, except sun-dried rhizome (CX-D5), contents of three major ingredients 4-6 decreased significantly, whereas, 1, 2, 8 and 9 increased significantly in all dried samples (Table 2). Moreover, contents of $\mathbf{1}$ and $\mathbf{7}$ in all dried samples were markedly higher and significantly lower than those in the fresh sample, respectively. The results indicated that all three major components and compound 7 degraded when fresh samples were dried by the two different methods examined.

Furthermore, using the same drying method, degradation extent of $\mathbf{5}$ and $\mathbf{6}$ was obviously greater when rhizomes were cut into slices. Whereas when slices were dried by different methods, similar patterns of chemical alterations were observed with no significant differences in overall degradation rates, although degree of alteration of individual components varied markedly. Moreover, the total content of $\mathbf{1}-\mathbf{9}$ in sundried rhizome (CX-D5) was similar to that of the fresh sample, while such content was significantly lower in other three dried samples. The results indicated that except sun-drying whole rhizomes, the most common and traditional way to dry Chuanxiong, other drying methods, such as heating whole rhizomes or sliced samples at $60^{\circ} \mathrm{C}$ in oven and sun-drying sliced samples, might cause significant alteration of main components, especially the three major components, in fresh 


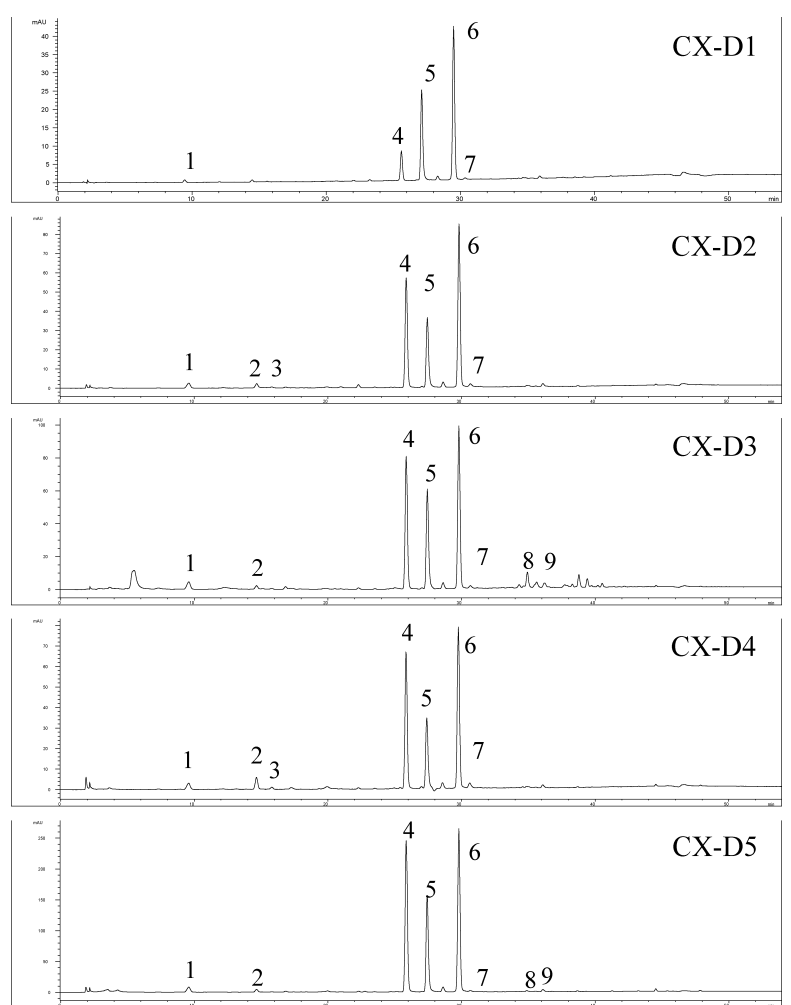

Fig. 5. Chromatograms of the Extracts Obtained from Rhizoma Chuanxiong Samples Dried Using Different Methods
Chuanxiong herb.

Influences of Processing Methods on the Main Components in Chuanxiong As shown in Table 3, compared with the dried but unprocessed sample (CX-D2), all three processing methods caused alteration of chemical profiles. The total content of nine constituents quantified in the stir-frying alone sample (CX-D2-P1) did not significantly change, while contents of two major compounds 5 and $\mathbf{6}$ decreased significantly leading to increases in contents of their corresponding degradation products $1,2,3,8$ and 9 . The total content of the main components in the bran-frying sample (CX-D2-P2) was about a half of that in unprocessed dried sample. The significant lose (about 98\%) was compound $\mathbf{5}$, while significant decreases in 4 and 6 and increases in 1, 2, 3, 8 and 9 were also observed. The reason for the significant decrease in the total content of main components is mainly due to the adsorption of lipophilic components in Chuanxiong by bran. Bran is a commonly used processing adjuvant to evenly heat herbal materials and to adsorb lipophilic compounds, which may cause irritation and/or other unwanted effects of TCM herbs. ${ }^{13)}$ In the case of wine-pretreated stir-frying sample (CX-D2-P3), the overall chemical profile did not change significantly, although as expected, there was a slight conversion of major compounds 5 and 6 to compounds 1, 2, 3, 8 and 9 . These results demonstrated that processing methods have different effects on chemical profiles of Chuanxiong.

In summary, post-harvest drying and processing procedures could significantly alter chemical profiles of Chuanxiong herb. In general, contents of three major components $\mathbf{4}$,

Table 2. Effects of Drying Procedure on the Contents of Nine Main Components in Chuanxiong

\begin{tabular}{|c|c|c|c|c|c|}
\hline \multirow{2}{*}{ Compound } & \multicolumn{5}{|c|}{ Content (mg/g of dried material) } \\
\hline & CX-D1 & CX-D2 & CX-D3 & CX-D4 & CX-D5 \\
\hline 1 & $0.16 \pm 0.03$ & $0.21 \pm 0.03$ & $0.28 \pm 0.03 * *$ & $0.33 \pm 0.03 * *$ & $0.25 \pm 0.04^{*}$ \\
\hline 2 & - & $0.09 \pm 0.01$ & $0.04 \pm 0.01$ & $0.32 \pm 0.04$ & $0.08 \pm 0.01$ \\
\hline 3 & - & - & - & $0.06 \pm 0.02$ & - \\
\hline 4 & $12.91 \pm 0.15$ & $8.34 \pm 0.91 * * *$ & $7.62 \pm 1.11 * * *$ & $10.72 \pm 0.27 * * *$ & $12.96 \pm 2.14$ \\
\hline 5 & $10.11 \pm 0.29$ & $5.06 \pm 0.43 * * *$ & $5.96 \pm 0.76^{* * *}$ & $6.05 \pm 0.49 * * *$ & $8.19 \pm 0.67 * *$ \\
\hline 6 & $13.42 \pm 0.52$ & $9.89 \pm 0.47 * *$ & $8.36 \pm 1.26^{* *}$ & $11.06 \pm 0.72 * *$ & $12.47 \pm 0.79$ \\
\hline 7 & $0.41 \pm 0.005$ & $0.20 \pm 0.01 * * *$ & $0.16 \pm 0.02 * * *$ & $0.36 \pm 0.02 * *$ & $0.10 \pm 0.01 * * *$ \\
\hline 8 & - & - & $0.29 \pm 0.01$ & $0.07 \pm 0.12$ & $0.03 \pm 0.001$ \\
\hline 9 & - & $0.08 \pm 0.01$ & $0.15 \pm 0.02$ & $0.13 \pm 0.08$ & $0.09 \pm 0.005$ \\
\hline Total $^{a)}$ & $37.48 \pm 0.78$ & $24.17 \pm 1.71 * * *$ & $23.22 \pm 3.20 * * *$ & $29.45 \pm 1.37 * * *$ & $34.56 \pm 3.53$ \\
\hline
\end{tabular}

a) Total content of all nine components measured. $* p<0.05, * * p<0.01, * * * p<0.001$ compared with the lyophilized sample CX-D1.

Table 3. Effects of Processing Procedure on the Contents of Nine Main Components in Chuanxiong

\begin{tabular}{|c|c|c|c|c|}
\hline \multirow{2}{*}{ Compound } & \multicolumn{4}{|c|}{ Content (mg/g of dried material) } \\
\hline & $\mathrm{CX}-\mathrm{D} 2$ & CX-D2-P1 & CX-D2-P2 & CX-D2-P3 \\
\hline 1 & $0.21 \pm 0.025$ & $0.44 \pm 0.022 * * *$ & $0.35 \pm 0.013 * * *$ & $0.36 \pm 0.05 * *$ \\
\hline 2 & $0.09 \pm 0.011$ & $0.15 \pm 0.006^{* * *}$ & $0.13 \pm 0.008^{* *}$ & $0.08 \pm 0.01$ \\
\hline 3 & - & $0.03 \pm 0.002$ & $0.03 \pm 0.002$ & $0.01 \pm 0.001$ \\
\hline 4 & $8.34 \pm 0.914$ & $8.57 \pm 0.374$ & $4.87 \pm 0.246^{* *}$ & $7.52 \pm 1.05$ \\
\hline 5 & $5.06 \pm 0.428$ & $4.21 \pm 0.084^{*}$ & $0.33 \pm 0.023 * * *$ & $4.53 \pm 0.59$ \\
\hline 6 & $9.89 \pm 0.472$ & $8.51 \pm 0.346^{* *}$ & $6.27 \pm 0.183^{* * *}$ & $9.57 \pm 0.88$ \\
\hline 7 & $0.20 \pm 0.009$ & $0.17 \pm 0.010^{* *}$ & $0.22 \pm 0.007$ & $0.16 \pm 0.02 *$ \\
\hline 8 & - & $0.06 \pm 0.001$ & $0.10 \pm 0.021$ & $0.04 \pm 0.01$ \\
\hline 9 & $0.08 \pm 0.006$ & $0.16 \pm 0.001 * * *$ & $0.20 \pm 0.015 * * *$ & $0.10 \pm 0.01 *$ \\
\hline Total $^{a)}$ & $24.17 \pm 1.705$ & $22.47 \pm 0.776$ & $12.63 \pm 0.479 * * *$ & $22.57 \pm 2.48$ \\
\hline
\end{tabular}

a) Total content of all nine components measured. $* p<0.05, * * p<0.01, * * * p<0.001$ compared with the unprocessed sample CX-D2. 
5 , and 6 decreased, while contents of $1,2,3,8$ and 9 increased when the herb underwent a drying and a processing process involving elevated temperature and/or sunlight exposure. Extensive hydrolysis of $\mathbf{5}$ to generate $\mathbf{1}$ occurred at higher temperature and sunlight exposure. At an elevated temperature $\mathbf{6}$ was mainly hydroxylated to generate $\mathbf{2}$ and $\mathbf{3}$, when exposed to sunlight, $\mathbf{6}$ underwent dimerization to form mainly 8 and 9. Different drying and processing methods caused different degrees of chemical conversion of main ingredients in Chuanxiong herb. As various ingredients, including $1,4,6,7,8$ and 9 , have been reported to be biologically active, ${ }^{4-9)}$ drying and processing methods caused variations in chemical profiles and quantities of the main bioactive components in Chuanxiong will inevitably alter therapeutic outcomes of the herb. Therefore, prior to the establishment of well-controlled and standardized drying and processing procedures, study of chemical and bioactive correlation should be conducted to reveal the extents of contribution of chemical profile alteration to the activity of Chuanxiong herb.

Acknowledgements The authors gratefully acknowledge the financial support of ITF fund (UIM/34) from Innovative Technology Council of The Hong Kong SAR Government. The authors also thank Tianfu Chuanxiong Pharmaceutical Development Company Ltd. for the fresh Chuanxiong samples.

\section{References}

1) Li S. L., Chan S. S. K., Lin G., Ling L., Yan R., Chung H. S., Tam Y. K., Planta Med., 69, 445-451 (2003).
2) Naito T., Sakata M., Ikeya Y., Okada M., Maruno M., Nat. Med., 49, 425-430 (1995).

3) Yan R., Li S. L., Chung H. S., Tam Y. K., Lin G., J. Pharm. Biomed. Anal., 37, 87-95 (2005).

4) Ko W. C., Liao C. C., Shih C. H., Lei C. B., Chen C. M., Planta Med., 68, 1004-1009 (2002)

5) Ko W. C., Sheu J. R., Tzeng S. H., Chen C. M., Planta Med., 64, 229-232 (1998).

6) Naito T., Kubota K., Shimoda Y., Sato T., Ikeya Y., Okada M., Nat. Med., 49, 288-292 (1995).

7) Teng C. M., Chen W. Y., Ko W. C., Ouyang C., Biochim. Biophys. Acta, 924, 375-382 (1987).

8) Wang P. S., Zhongguo Yiyao Gongye, 19, 553-559 (1988).

9) Chan S. S. K., Choi A. O. K., Jones R. L., Lin G., Eur. J. Pharmacol., 537, 111-117 (2006).

10) Li S. L., Lin G., Chung H. S., Tam Y. K., Acta Pharm. Sin., 39, 621626 (2004).

11) Li S. L., Lin G., Tam Y. K., Planta Med., 72, 278-280 (2006).

12) The State Pharmacopoeia Commission of People's Republic of China, "Pharmacopoeia of the People's Republic of China," Part I (2005 edition), Chemical Industry Press, Beijing, 2005, p. 37.

13) Ye D. J., Zhang S. C., Chen Q., "Processing of Traditional Chinese Medicine," Shanghai Science \& Technology Press, Shanghai, 1998.

14) Lin L. Z., He X. G., Lian L. Z., King W., Elliott J., J. Chromatogr. A, 810, 71-79 (1998).

15) Kobayashi M., Fujita M., Mitsuhashi H., Chem. Pharm. Bull., 32, 3770-3773 (1984).

16) Kobayashi M., Fujita M., Mitsuhashi H., Chem. Pharm. Bull., 35, 1427-1433 (1987).

17) Lu G. H., Chan K., Leung K., Chan C. L., Zhao Z. Z., Jiang Z. H., J. Chromatogr. A, 1068, 209-219 (2005).

18) Cui F., Feng L., Hu J., Drug Dev. Ind. Pharm., 32, 747-755 (2006). 